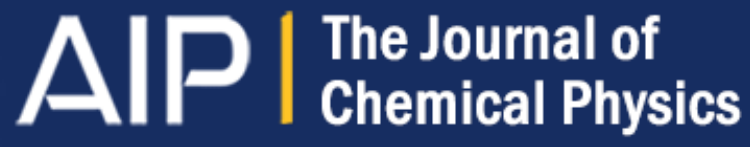

Rotational branching ratios and photoelectron angular distributions in resonance enhanced multiphoton ionization of diatomic molecules

Kwanghsi Wang and V. McKoy

Citation: The Journal of Chemical Physics 95, 4977 (1991); doi: 10.1063/1.461793

View online: http://dx.doi.org/10.1063/1.461793

View Table of Contents: http://scitation.aip.org/content/aip/journal/jcp/95/7?ver=pdfcov

Published by the AIP Publishing

\section{Articles you may be interested in}

Molecular-orbital decomposition of the ionization continuum for a diatomic molecule by angle- and energyresolved photoelectron spectroscopy. I. Formalism

J. Chem. Phys. 104, 4554 (1996); 10.1063/1.471204

Complex angular momentum theory of molecular collisions: New phase rules for rotationally inelastic diffraction scattering in atom-homonuclear diatomic molecule collisions

J. Chem. Phys. 98, 2947 (1993); 10.1063/1.464123

Rotational branching ratios and photoelectron angular distributions in resonance enhanced multiphoton ionization of $\mathrm{HBr}$ via the $\mathrm{F} 1 \Delta 2$ Rydberg state

J. Chem. Phys. 95, 7872 (1991); 10.1063/1.461316

Rotationally resolved photoelectron angular distributions in resonance enhanced multiphoton ionization of NO

J. Chem. Phys. 91, 2235 (1989); 10.1063/1.457031

Rotational branching ratios at low photoelectron energies in resonant enhanced multiphoton ionization of NO

J. Chem. Phys. 90, 2570 (1989); 10.1063/1.455953

\section{AIP $\left.\right|_{\text {APL Photonics }}$}

APL Photonics is pleased to announce Benjamin Eggleton as its Editor-in-Chief

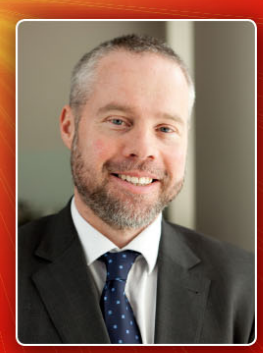




\title{
Rotational branching ratios and photoelectron angular distributions in resonance enhanced multiphoton ionization of diatomic molecules
}

\author{
Kwanghsi Wang and V. McKoy \\ Arthur Amos Noyes Laboratory of Chemical Physics, California Institute of Technology, Pasadena, \\ California $91125^{\mathrm{a}}$
}

(Received 2 May 1991; accepted 17 June 1991)

\begin{abstract}
In this paper we extend a previous formulation of molecular resonance enhanced multiphoton ionization (REMPI) photoelectron spectra to explicitly include multiplet-specific final state wave functions and intermediate coupling schemes. The results of this formulation should be well suited and helpful in quantitative theoretical studies of rotationally resolved REMPI spectra in many diatomic molecules of interest. As an example, we use this formulation to study the rotational branching ratios and photoelectron angular distributions for $(3+1)$ REMPI of NH via the $3{ }^{3} \Pi$ Rydberg resonant state. The predicted anomalous rotational distributions are interpreted as arising from a Cooper minimum in the $l=2$ component of the $k \pi$ photoionization channel. A number of other results are obtained and discussed.
\end{abstract}

\section{INTRODUCTION}

Resonance enhanced multiphoton ionization (REMPI) has been used to obtain detailed spectroscopic information ${ }^{1}$ for molecules and, combined with high-resolution photoelectron spectroscopy (PES), to study branching ratios and photoelectron angular distributions for photoionization of individual rovibrational levels of excited states of molecules. Such rotational and vibrational ion distributions are important not only for our understanding of the dynamics of molecular photoionization at a quantum-state-specific level but they can also be exploited to prepare ions in a stateselective manner for subsequent studies. Several rotationally selective experiments have been performed on $\mathrm{H}_{2},{ }^{2-7}$ $\mathrm{D}_{2},{ }^{8-10}$ and on high $J$ states of NO, ${ }^{11-14}$ where the rotational spacing is large enough to allow rotational resolution in the photoelectron spectra. Zero-kinetic-energy (ZEKE) photoionization spectroscopy has also been used to obtain ion rotational spectra for very low $J$ states. ${ }^{15,16}$ These experiments reveal interesting spectral features such as the dependence of photoelectron angular distributions on rotational levels, ${ }^{7.14}$ rotational propensity rules, ${ }^{12-15.17}$ parity selectivity in transitions between electronically degenerate states, ${ }^{3,4,12,17.18}$ effects of alignment, ${ }^{5}$ and autoionization. ${ }^{6,10}$ Xie and Zare ${ }^{18}$ and Fujii et al. ${ }^{17}$ have also directly observed rotational ion distributions via laser induced fluorescence spectra (LIF) of $\mathrm{HBr}^{+}$and $\mathrm{N}_{2}^{+}$, respectively. Other diatomic molecules such as light hydrides are good candidates for rotationally resolved studies in the near future.

Buckingham et al. ${ }^{19,20}$ first presented general expressions governing rotationally resolved photoelectron spectra. Later, Fano and Dill ${ }^{21}$ and Dill ${ }^{22}$ used multichannel quantum defect theory ${ }^{23,24}$ to determine rotationally resolved cross sections and angular distributions for $\mathrm{H}_{2}$. More recently, formulations including vibrational motion have been given by Itikawa ${ }^{25}$ and Chandra. ${ }^{26}$ Dixit et al. ${ }^{27,28}$ have also developed a final state wave function treatment of rotation-

\footnotetext{
") Contribution No. 8435 .
}

ally resolved photoelectron spectra in the context of REMPI applications, and presented rotational propensity ${ }^{27}$ and parity ${ }^{28}$ selection rules. This formulation has been used in studies of rotational branching ratios and photoelectron angular distributions in REMPI of NO via the $A^{2} \Sigma+29,30$ $D^{2} \Sigma^{+},{ }^{30,31}$ and $C^{2} \Pi^{32}$ states and of the effects of orbital evolution on rotational branching ratios in $(2+1)$ REMPI of $\mathrm{CH} .{ }^{33}$ Similar parity selection rules were also obtained by Fredin et al..$^{34}$ and more recently by Xie and Zare. ${ }^{35}$

In this paper we extend a formulation ${ }^{27,28}$ for REMPI photoelectron spectra of diatomic molecules. A useful feature of this extension is that it explicitly assumes that multiplet-specific final state wave functions are used for the photoionized system. With such wave functions we present general expressions for the photoionization matrix elements leading to ions with total spin $S \neq 0$. We use intermediate coupling schemes to represent the resonant and ionic states which are appropriate for studies of states lying between pure Hund's cases (a) and (b). The formulation given here, with its inclusion of multiplet-specific final state wave functions and intermediate coupling schemes, should be well suited and helpful in quantitative theoretical studies of rotationally resolved REMPI spectra in a wide range of diatomic molecules, e.g., $\mathrm{HBr}, \mathrm{OH}, \mathrm{NH}, \mathrm{O}_{2}$, and NF. A formula for pure Hund's case (b) coupling scheme which generalizes the work of Dixit and $\mathrm{McKoy}^{28}$ to include the spin couplings of the final state wave functions is also given. The effect of alignment is also included throughout the formulation.

As an example, this formulation is used here to study the rotational branching ratios and photoelectron angular distributions associated with $\Delta v=v_{+}-v_{i}=0,1$ vibrational transitions for $(3+1)$ REMPI of NH via the $3{ }^{3}$ II state. We choose this specific example for the following reasons. Experimental studies of the rotationally resolved REMPI spectra are under way. ${ }^{36}$ Previous theoretical studies of vibrationally resolved REMPI spectra have shown clear evidence for the presence of a Cooper minimum in this system. ${ }^{37}$ The resulting ion distributions can hence be expected to show the unusual and striking behavior similar to that seen recently in the REMPI spectra of $\mathrm{OH}$ and which can be explained quan- 
titatively as arising from a Cooper minimum. ${ }^{38}$ Furthermore, this system provides a good example of the usefulness of the intermediate coupling scheme in describing ${ }^{3} \Pi \rightarrow^{2} \Pi$ transitions.

\section{FORMULATION}

In an $(n+1)$ REMPI process of interest here, the resonant intermediate state $|i\rangle$ is created by absorption of $n$ photons of frequency $\omega$ from the initial state $|0\rangle$ of a molecule. This state is subsequently ionized by an additional photon, i.e.,

$$
A B \rightarrow A B^{*} \stackrel{\hbar \omega^{\prime}}{\rightarrow} A B^{+}+e(\mathbf{k}) .
$$

The kinetic energy and wave vector of the photoelectron are denoted by $\epsilon$ and $\mathbf{k}$, respectively (i.e., $\epsilon=k^{2} / 2$ ). The frequency $\omega^{\prime}$ differs from $\omega$ in a two-color study. For linearly polarized light, ionization out of each $M_{J}$ magnetic sublevel of the initial state forms an independent channel. In the perturbative limit, the differential cross section for $(n+1)$ photon ionization is given by

$$
\frac{d \sigma}{d \Omega} \propto \sum_{M_{0}}\left|\frac{\left\langle f\left|D_{\mu_{0}}\right| i\right\rangle\left\langle i\left|D_{n}\right| 0\right\rangle}{E_{i}-E_{0}-n \hbar \omega+i \Gamma_{i}}\right|^{2} .
$$

In this equation, $D_{\mu_{0}}$ denotes the electronic dipole moment operator, $D_{n}$ the effective dipole moment operator for $n$ photon excitation, $\mu_{0}$ the light polarization index in the laboratory frame, $\Gamma_{i}$ the width of state $|i\rangle,|f\rangle$ the final continuum state of the (ion + photoelectron) system, and $E_{i}$ the energy of state $|i\rangle$. Furthermore, for the branching ratios of interest here, the constant implied in Eq. (2) is unimportant and will be suppressed.

The effective bound-bound dipole matrix element for $n$ photon absorption can be written as

$$
\left\langle i\left|D_{n}\right| 0\right\rangle=\left\langle\gamma_{i} J_{i} M_{J_{i}}\left|D_{n}\right| \gamma_{0} J_{0} M_{J_{0}}\right\rangle=R_{n}\left\langle\gamma_{i} J_{i}\|D\| \gamma_{0} J_{0}\right\rangle
$$

with

$$
D_{n}=D_{\mu_{0}}\left(\sum_{p} \prod_{k=1}^{n-1} \frac{\left|\gamma_{k} J_{k} M_{J_{k}}\right\rangle_{p p}\left\langle\gamma_{k} J_{k} M_{J_{k}}\right| D_{\mu_{0}}}{E_{k}-E_{0}-k \hbar \omega+i \Gamma_{k}}\right)
$$

where $p$ indicates a summation over all possible paths, and $\gamma_{0}, \gamma_{i}$, and $\gamma_{k}$ represent all other quantum numbers required for an unambiguous designation of states $|0\rangle,|i\rangle$, and $|k\rangle$, respectively. Here $|k\rangle \equiv\left|\gamma_{k} J_{k} M_{J_{k}}\right\rangle$ includes all dipole-allowed virtual states in the first $n$-photon absorption step. The rotational part of the dipole moment $R_{n}$ is independent of the coupling schemes used for representing the states $|0\rangle$, $|i\rangle$, and $|k\rangle$, and has the form

$$
R_{n}=\prod_{k=1}^{n}(-1)^{J_{k}-M_{J_{k}}}\left(\begin{array}{ccc}
J_{k} & 1 & J_{k-1} \\
-M_{J_{k}} & \mu_{0} & M_{J_{k-1}}
\end{array}\right) .
$$

Properties of these $3-j$ symbols lead to the condition $\Delta M=M_{J_{k}}-M_{J_{k-1}}=\mu_{0}$. The evaluation of the reduced matrix element $\left\langle\gamma_{i} J_{i}\|D\| \gamma_{0} J_{0}\right\rangle$ requires a summation over all possible paths and virtual states. In many cases explicit evaluation of these summations is not necessary, if one is interested only in the relative populations (the alignment) of the different $M_{J}$ levels of the resonant state. Under these circumstances, $\left\langle\gamma_{i} J_{i}\|D\| \gamma_{0} J_{0}\right\rangle$ is just an overall multiplicative factor. For other cases $\left\langle\gamma_{i} J_{i}\|D\| \gamma_{0} J_{0}\right\rangle$ must be explicitly evaluated. $\left|\left\langle\gamma_{i} J_{i}\|D\| \gamma_{0} J_{0}\right\rangle\right|^{2}$ is the rotational line strength which is available from Ref. 39 for two and threephoton transitions in diatomic molecules. Note that Eq. (5) reduces to the formula of Dixit et al. ${ }^{27}$ for one-photon excitation.

For a specific $J_{0} \rightarrow J_{i}$ transition (on a given branch), dipole selection rules ${ }^{40}$ govern which quantum state specific level (e.g.,,,+- or $c, d$, or $e, f$ ) of the virtual and resonant rovibronic states can be accessed. Here we adopt Kronig states that are eigenstates of the $\hat{\sigma}_{v}$ operator. For diatomic molecules, the Kronig symmetry operator is just the $\hat{\sigma}_{v}$ symmetry operator. We also find that the $c / d$ notation is convenient in the formulation. Following Mizushima, ${ }^{41}$ Hund's case (a) states with $\hat{\sigma}_{v}$ symmetry properties can be defined as

$$
\begin{aligned}
\left|\gamma_{i} p_{i} q_{i} \bar{\Lambda}_{i} S_{i} \Sigma_{i} J_{i} \Omega_{i} M_{J_{i}}\right\rangle & \\
= & \frac{1}{\sqrt{2}}\left[\left|\gamma_{i} \bar{\Lambda}_{i} S_{i} \Sigma_{i} J_{i} \Omega_{i} M_{J_{i}}\right\rangle\right. \\
& \left.+(-1)^{p_{i}+q_{i}}\left|\gamma_{i}-\bar{\Lambda}_{i} S_{i}-\Sigma_{i} J_{i}-\Omega_{i} M_{J_{i}}\right\rangle\right],
\end{aligned}
$$

where

$$
\begin{aligned}
& \bar{\Lambda}_{i}=\left|\Lambda_{i}\right|, \\
& p_{i}= \begin{cases}0 & \text { for } c \text { states } \\
1 & \text { for } d \text { states }\end{cases}
\end{aligned}
$$

and

$$
q_{i}= \begin{cases}1 & \text { for } \Sigma^{-} \text {states } \\ 0 & \text { for others }\end{cases}
$$

As usual, in the above equation $\Omega_{i}$ denotes the total electronic angular momentum about the internuclear axis, $\Lambda_{i}$ the projection of electronic orbital angular momentum along the internuclear axis, $S_{i}$ the total spin, $\Sigma_{i}$ its projection along the internuclear axis, $J_{i}$ the total angular momentum, and $M_{J_{i}}$ its projection along the laboratory $z$ axis. Note that for $\Lambda=\Omega=0$, there is no $d$ state of $\Sigma^{+}$symmetry or $c$ state of $\Sigma^{-}$symmetry. For Hund's case (a) the phase factor of the Kronig state is given by

$$
\begin{aligned}
& \hat{\sigma}_{v}\left|\gamma_{i} p_{i} q_{i} \bar{\Lambda}_{i} S_{i} \Sigma_{i} J_{i} \Omega_{i} M_{J_{i}}\right\rangle \\
& \quad=(-1)^{J_{i}+p_{i}-s_{i}}\left|\gamma_{i} p_{i} q_{i} \bar{\Lambda}_{i} S_{i} \Sigma_{i} J_{i} \Omega_{i} M_{J_{i}}\right\rangle .
\end{aligned}
$$

In the $e / f$ notation, ${ }^{42}$ a $c$ level is equivalent to an $e$ level for even $\left(T_{i}-S_{i}\right)$ and to an $f$ level for odd $\left(T_{i}-S_{i}\right)$ with $T_{i}=0$ for molecules with an even number of electrons, and $\frac{1}{2}$ for molecules with an odd number of electrons. Similarly, a $d$ level is equivalent to an $e$ level for odd $T_{i}-S_{i}$ and to an $f$ level for even $T_{i}-S_{i}$. However, with Hund's case (b) basis states $\left(T_{i}+\Sigma_{i}\right)$ determines the relationship between $c / d$ and $e / f$ as $\left(T_{i}-S_{i}\right)$ does for Hund's case (a) basis states.

In these studies we use the intermediate coupling scheme to evaluate the matrix element $\left\langle f_{\beta}\left|D_{\mu_{0}}\right| i_{\alpha}\right\rangle$ for photoionization of a parity level $\alpha$ of the resonant state leading to a parity level $\beta$ in the ion. The intermediate coupling scheme is suitable for any Hund's case and uses Hund's case (a) 
basis states. Following Hougen, ${ }^{43}$ the rovibronic state $\left|\Lambda S \Sigma ; J \Omega M_{J}\right\rangle$ associated with a particular electronic-vibrational state is a solution of the Schrödinger equation

$$
\hat{H}\left|\Lambda S \Sigma ; J \Omega M_{J}\right\rangle=E\left|\Lambda S \Sigma ; J \Omega M_{J}\right\rangle,
$$

with

$$
\widehat{H}=\hat{H}_{e v}+\widehat{H}_{r},
$$

where $\widehat{H}_{e v}$ and $\hat{H}_{r}$ are the electronic-vibrational and rotational parts of the Hamiltonian, respectively, and $E$ the energy of the eigenstate. Neglecting $B\left\langle L_{1}^{2}\right\rangle$, for diatomic molecules, $\widehat{H}_{e v}$ and $\widehat{H}_{r}$ have the following forms:

$$
\hat{H}_{e v}=\hat{H}_{e v}^{0}+A L_{z} S_{z} \text {, }
$$

and

$\hat{H}_{r}=B\left(J^{2}-J_{z}^{2}+S^{2}-S_{z}^{2}\right)-B\left(J_{+} S_{-}+J_{-} S_{+}\right)$,

where $A$ and $B$ are the spin-orbit splitting and rotational constants, respectively. $\widehat{H}_{e v}^{\circ}$, which contributes a constant energy only, is the electronic-vibrational Hamiltonian without the spin-orbit interaction. The cross terms $J_{+} S_{-}$and $J_{-} S_{+}$in Eq. (12) mix pure-case (a) and (b) states and thus give rise to the intermediate coupling scheme state notation $\left|\Lambda S \Sigma ; J \Omega M_{J}\right\rangle$.

Diagonalization of the $(2 S+1) \times(2 S+1)$ Hamiltonian $\hat{H}$ in this $(2 S+1)$ basis yields eigenvalues and corresponding eigenvectors. We denote the set of rovibronic states as $\left|F_{n}\right\rangle, n=1,2, \ldots,(2 S+1)$

$$
\left|F_{n}\right\rangle=\sum_{j=1}^{2 S+1} C_{j n}\left|\Lambda S \Sigma_{j} ; J \Omega_{j} M_{J}\right\rangle
$$

The $F_{n}$ indices label the energy ordering of the eigenvalues for a given $J$, with $n=1$ the lowest and $n=2 S+1$ the highest. In the limiting cases $A \sim 0$ and $A / B \sim \infty$, this treatment reduces to that of pure Hund's cases (b) and (a), respectively. Also, from Eq. (6), the Kronig component $\alpha$ of the perturbed rovibronic state is given by

$$
\begin{aligned}
\left|F_{n \alpha}\right\rangle= & \sum_{j} \frac{C_{j n}}{\sqrt{2}}\left[\left|\bar{\Lambda} S \Sigma_{j} J \Omega_{j} M_{J}\right\rangle+(-1)^{p+q}\right. \\
& \left.\times\left|-\bar{\Lambda} S-\Sigma_{j} J-\Omega_{j} M_{J}\right\rangle\right],
\end{aligned}
$$

where $\alpha=c$ or $d$ (or $e$ or $f$ ) and $p, q$ are defined in Eq. (7). The state indices $i$ and + for the resonant and ionic states are omitted in Eqs. (13) and (14).

We now examine the photoionization matrix element for ionization of a resonant state with Kronig symmetry $\alpha$ of a rovibronic state $\left|F_{n_{i}}\right\rangle$ of state $|i\rangle$ to a final continuum state with Kronig symmetry $\beta$ of a rovibronic eigenstate $\left|F_{n_{+}}\right\rangle$of state $\left|f^{+}\right\rangle$. The bound-free transition moment can be written as

$$
\begin{aligned}
\left\langle f_{\beta}\left|D_{\mu_{0}}\right| i_{\alpha}\right\rangle= & \sum_{\Lambda_{f^{2}} f}\left\langle\phi_{e}\right|\left\langle F_{n_{+} \beta} \mid \Lambda_{f} \Sigma_{f}\right\rangle\left\langle\Lambda_{f} \Sigma_{f}\left|D_{\mu_{0}}\right| F_{n_{f} \alpha}\right\rangle \\
= & \sum_{\substack{\Lambda_{f_{f}} \Sigma_{f} \\
n_{+}^{\prime} n_{i}^{\prime}}} \frac{C_{n_{+}^{\prime} n_{+}} C_{n_{i}^{\prime} n_{i}}}{2}\left(I_{1}+I_{2}+I_{3}+I_{4}\right),
\end{aligned}
$$

$$
\begin{aligned}
I_{1}= & \left\langle\phi_{e}\right|\left\langle\gamma_{+} \bar{\Lambda}_{+} S_{+} \Sigma_{+, n_{+}^{\prime}} M_{J_{+}} \mid \Lambda_{f} \Sigma_{f}\right\rangle \\
& \times\left\langle\Lambda_{f} \Sigma_{f}\left|D_{\mu_{0}}\right| \gamma_{i} \bar{\Lambda}_{i} S_{i} \Sigma_{i, n_{i}^{\prime}} J_{i} \Omega_{i, n_{i}^{\prime}} M_{J_{i}}\right\rangle \\
I_{2}= & (-1)^{p_{+}+q_{i}} \\
& \times\left\langle\phi_{e}\right|\left\langle\gamma_{+} \bar{\Lambda}_{+} S_{+} \Sigma_{+, n_{+}^{\prime}} J_{+} \Omega_{+, n_{+}^{\prime}} M_{J_{+}} \mid \Lambda_{f} \Sigma_{f}\right\rangle \\
& \times\left\langle\Lambda_{f} \Sigma_{f}\left|D_{\mu_{0}}\right| \gamma_{i}-\bar{\Lambda}_{i} S_{i}-\Sigma_{i, n_{i}^{\prime}} J_{i}-\Omega_{i, n_{i}^{\prime}} M_{J^{\prime}}\right\rangle, \\
I_{3}= & (-1)^{p_{+}+q_{+}}\left\langle\phi_{e}\right| \\
& \times\left\langle\gamma_{+}-\bar{\Lambda}_{+} S_{+}-\Sigma_{+, n_{+}^{\prime}} J_{+}\right. \\
& -\Omega_{+, n_{+}^{\prime}} M_{J_{+}}\left|\Lambda_{f} \Sigma_{f}\right\rangle \\
& \times\left\langle\Lambda_{f} \Sigma_{f}\left|D_{\mu_{0}}\right| \gamma_{i} \bar{\Lambda}_{i} S_{i} \Sigma_{i, n_{i}^{\prime}} J_{i} \Omega_{i, n_{i}^{\prime}} M_{J_{i}}\right\rangle
\end{aligned}
$$

and

$$
\begin{aligned}
I_{4}= & (-1)^{p_{i}+q_{i}+p_{+}+q_{+}}\left\langle\phi_{e}\right| \\
& \times\left\langle\gamma_{+}-\bar{\Lambda}_{+} S_{+}-\Sigma_{+, n_{+}^{\prime}} J_{+}-\Omega_{+, n_{+}^{\prime}} M_{J} \mid \Lambda_{f} \Sigma_{f}\right\rangle \\
& \times\left\langle\Lambda_{f} \Sigma_{f}\left|D_{\mu_{0}}\right| \gamma_{i}-\bar{\Lambda}_{i} S_{i}-\Sigma_{i, n_{f}^{\prime}} J_{i}-\Omega_{i, n_{i}^{\prime}} M_{j}\right\rangle, \quad(16 \mathrm{~d})
\end{aligned}
$$

where $C_{n_{+}^{\prime} n_{+}}$and $C_{n_{i}^{\prime} n_{i}}$ are coefficients of perturbed rovibronic states of the ion and resonant states, respectively, and $\left|\Lambda_{f} \Sigma_{f}\right\rangle$ are the multiplet-specific final state wave functions including spin. $\left|\Lambda_{f} \Sigma_{f}\right\rangle$ are antisymmetrized products of the photoelectron and ion wave functions which may consist of several spin combinations as

$$
\left|\Lambda_{f} \Sigma_{f}\right\rangle=\sum_{m} C_{m}\left|\Lambda_{+} S_{+} \Sigma_{+} J_{+} \Omega_{+} M_{J_{+}} ; l_{e} M_{l_{e}} S_{e} \Sigma_{e}\right\rangle,
$$

with $S_{e}=\frac{1}{2}$ and $\Sigma_{e}= \pm \frac{1}{2}$ for the photoelectron, and $C_{m}$ is an expansion coefficient. In Eq. (15) the total final state continuum wave function

$$
|f\rangle=\sum_{\Lambda_{f_{f}}}\left|\Lambda_{f} \Sigma_{f}\right\rangle\left\langle\Lambda_{f} \Sigma_{f} \mid f\right\rangle,
$$

is used. In Eqs. (15) and (16) $\left|\phi_{e}\right\rangle$ is the photoelectron wave function given by

$$
\begin{aligned}
\left|\phi_{e}\right\rangle= & \sum_{\Sigma_{e}}(-1)^{\Sigma_{e}-m_{e}} \mathscr{D}_{m_{e} \Sigma_{e}}^{S_{e}}\left|S_{e} \Sigma_{e}\right\rangle \sum_{\lambda \lambda m} i^{l} e^{-i \eta_{l}}(-1)^{m-\lambda} \\
& \times Y_{l m}^{*}(\hat{k}) \mathscr{D}_{m \lambda}^{l} \psi_{k l \lambda}
\end{aligned}
$$

in the molecular frame. Here $\mathscr{D}_{m \lambda}^{l}$ are rotational matrices in Edmonds's notation, ${ }^{44} \eta_{l}$ the Coulomb phase shift, $\psi_{k l \lambda}$ a partial wave component of the photoelectron orbital ${ }^{45,46}$ for momentum k. In Eq. (19) the photoelectron wave function is expanded in spherical harmonics $Y_{l m}(\hat{k})$. Below we will suppress the subindices $n_{+}^{\prime}$ and $n_{i}^{\prime}$ used in Eq. (16).

With the following molecular frame basis for both the ionic state and bound state wave functions

$$
\begin{aligned}
\left|\gamma_{+} \Lambda_{+} S_{+} \Sigma_{+} J_{+} \Omega_{+} M_{J_{+}}\right\rangle \\
=\sqrt{\frac{2 J_{+}+1}{8 \pi^{2}}} \psi_{\gamma_{+} \Lambda_{+}}^{e}(\mathrm{r} ; R) \chi_{v_{+}}(R) \\
\quad \times(-1)^{M_{J_{+}}-\Omega_{+} \mathscr{D}_{M_{J_{+}} \Omega_{+}}^{J_{+}}(R)\left|S_{+} \Sigma_{+}\right\rangle,}
\end{aligned}
$$




$$
\begin{aligned}
\left|\gamma_{i} \Lambda_{i} S_{i} \Sigma_{i} J_{i} \Omega_{i} M_{J_{i}}\right\rangle= & \sqrt{\frac{2 J_{i}+1}{8 \pi^{2}}} \psi_{\gamma_{i} \Lambda_{i}}^{e}(\mathrm{r} ; R) \chi_{v_{i}}(R) \\
& \cdot(-1)^{M_{J_{i}}-\Omega_{i}} \mathscr{D}_{M_{J_{i}} \Omega_{i}}^{J_{i}}(R)\left|S_{i} \Sigma_{i}\right\rangle,
\end{aligned}
$$

and the dipole operator in the molecular frame

$$
D_{\mu_{0}}=\sqrt{\frac{4 \pi}{3}} r \sum_{\mu}(-1)^{\mu-\mu_{0}} \mathscr{D}_{\mu_{0} \mu}^{1} Y_{1 \mu}(\hat{r}),
$$

$I_{1}$ of Eq. (16a) can be written as

$$
\begin{aligned}
I_{1}= & \sqrt{\frac{4 \pi}{3}}\left[\left(2 J_{+}+1\right)\left(2 J_{i}+1\right)\left(2 S_{i}+1\right)\right]^{1 / 2} \\
& \times \sum_{\substack{J_{J} m_{\lambda} \lambda_{t} \\
J_{r} m_{l} l_{r} \\
m_{e} l m}} Q \tilde{I}_{1} Y_{l m}^{(\hat{k})},
\end{aligned}
$$

with

$$
\begin{aligned}
& Q=(-1)^{M_{J_{t}+S_{+}+1 / 2}}\left(2 J_{t}+1\right)\left(2 J_{r}+1\right) \\
& \times\left(\begin{array}{ccc}
J_{+} & J_{i} & J_{t} \\
-M_{J_{+}} & M_{J_{r}} & m_{t}
\end{array}\right)\left(\begin{array}{ccc}
S_{e} & l & J_{r} \\
-m_{e} & -m & m_{r}
\end{array}\right) \\
& \times\left(\begin{array}{ccc}
J_{r} & 1 & J_{t} \\
-m_{r} & \mu_{0} & -m_{t}
\end{array}\right), \\
& \tilde{I}_{1}=\sum_{\substack{\mu \lambda \\
\Sigma_{e}}} \tilde{I}_{L \lambda \mu_{i} \Sigma_{e}}(-1)^{-\Omega_{i}+\Sigma_{i}} G\left(\begin{array}{ccc}
J_{+} & J_{i} & J_{t} \\
-\Omega_{+} & \Omega_{i} & \lambda_{t}
\end{array}\right) \\
& \times\left(\begin{array}{ccc}
S_{+} & S_{e} & S_{i} \\
\Sigma_{+} & \Sigma_{e} & -\Sigma_{i}
\end{array}\right), \\
& \tilde{I}_{2 \lambda_{\mu ;} ; \Sigma_{e}}=\sum_{\Lambda_{f} \Sigma_{f}}\left\langle\Lambda_{+} \lambda \mid \Lambda_{f}\right\rangle\left\langle\Sigma_{+} \Sigma_{e} \mid \Sigma_{f}\right\rangle I_{l \lambda_{\mu}}\left(\Lambda_{f} \Sigma_{f}\right) \text {, }
\end{aligned}
$$

and

$G=\left(\begin{array}{ccc}J_{r} & 1 & J_{t} \\ -\lambda_{r} & \mu & -\lambda_{t}\end{array}\right)\left(\begin{array}{ccc}S_{e} & l & J_{r} \\ -\Sigma_{e} & -\lambda & \lambda_{r}\end{array}\right)$.

From properties of $3-j$ symbols, $J_{r}$ and $J_{t}$ are restricted by $\left|J_{t}-J_{r}\right| \leqslant 1$. In Eqs. (22), $l$ is the partial wave component of the photoelectron orbital and $m$ its magnetic quantum number. Equations (22b) and (22d) give the restriction

$$
\mu-\lambda= \pm\left(\bar{\Lambda}_{+}-\bar{\Lambda}_{i}\right)
$$

for the angular momentum transfer in the photoionization process. Note that the sign in Eq. (23) differs from that of
Lucchese et al. ${ }^{45}$ [cf. Eq. (47) of Ref. 45]. The $I_{l \lambda \mu}\left(\Lambda_{f} \Sigma_{f}\right)$ is the vibrationally averaged photoelectron matrix element between the resonant state and the photoelectron continuum wave function in the molecular frame

$$
I_{i \lambda \mu}=(-i)^{l} e^{i \eta_{l}} \int d R \chi_{v_{+}}^{*}(R) r_{f_{i}}^{l \lambda \mu}(R) \chi_{v_{i}}(R),
$$

where

$$
r_{f}^{l \lambda \mu}(R)=\left\langle\psi_{k l \lambda}(\mathbf{r}, R)\left|r Y_{1 \mu}\right| \psi_{i}(\mathbf{r}, R)\right\rangle .
$$

For some states of singlet spin $\tilde{I}_{i \mu ; \Sigma_{e}}\left(\Lambda_{f} \Sigma_{f}\right)$ reduces to $I_{l \lambda \mu}\left(\Lambda_{f} \Sigma_{f}\right)$. Equation (21) is then usually used to describe the rotational ion distribution for the Hund's case (a) when the parity of rotational levels is neglected. For linear molecules, we also have

$$
\tilde{I}_{l \lambda \mu ; \Sigma_{e}}\left(\Lambda_{f} \Sigma_{f}\right)=\tilde{I}_{l-\lambda-\mu ;-\Sigma_{e}}\left(\Lambda_{f} \Sigma_{f}\right) .
$$

We will refer to $\tilde{I}_{l \lambda \mu ; \Sigma_{e}}$ as a "generalized vibrationally averaged dipole moment" to distinguish it from $I_{i \lambda \mu}$.

Omitting the term $Q$ and the overall constant in Eq. (21) $, I_{2}, I_{3}$, and $I_{4}$ can be similarly shown to be given by

$$
\begin{aligned}
\tilde{I}_{2}= & \sum_{\substack{\mu \lambda \\
\Sigma_{e}}} \tilde{I}_{l \lambda \mu ; \Sigma_{e}}(-1)^{\Omega_{i}-\Sigma_{i}+p_{i}+q_{i}} G\left(\begin{array}{ccc}
J_{+} & J_{i} & J_{t} \\
-\Omega_{+} & -\Omega_{i} & \lambda_{t}
\end{array}\right) \\
& \times\left(\begin{array}{lll}
S_{+} & S_{e} & S_{i} \\
\Sigma_{+} & \Sigma_{e} & \Sigma_{i}
\end{array}\right), \\
\tilde{I}_{3}= & \sum_{\substack{\mu \lambda \\
\Sigma_{e}}} \tilde{I}_{i \lambda \mu ; \Sigma_{e}}(-1)^{-\Omega_{i}+\Sigma_{i}+p_{+}+q_{+}} G\left(\begin{array}{ccc}
J_{+} & J_{i} & J_{t} \\
\Omega_{+} & \Omega_{i} & \lambda_{t}
\end{array}\right) \\
& \times\left(\begin{array}{ccc}
S_{+} & S_{e} & S_{i} \\
-\Sigma_{+} & \Sigma_{e} & -\Sigma_{i}
\end{array}\right),
\end{aligned}
$$

and

$$
\begin{aligned}
\tilde{I}_{4}= & \sum_{\substack{\mu \lambda \\
\Sigma_{e}}} \tilde{I}_{l \lambda \mu ; \Sigma_{e}}(-1)^{\Omega_{i}-\Sigma_{i}+p_{i}+q_{i}+p_{+}+q_{+}} G \\
& \times\left(\begin{array}{cccc}
J_{+} & J_{i} & J_{t} \\
\Omega_{+} & -\Omega_{i} & \lambda_{t}
\end{array}\right)\left(\begin{array}{ccc}
S_{+} & S_{e} & S_{i} \\
-\Sigma_{+} & \Sigma_{e} & \Sigma_{i}
\end{array}\right) .
\end{aligned}
$$

By combining Eqs. (27) and (22b) with Eq. (26) and properties of the 3-j symbols, the bound-free transition moment, Eq. (15), can be expressed in terms of spherical harmonics $Y_{l m}(\hat{k})$ as

$$
\left\langle f_{\beta}\left|D_{\mu_{0}}\right| i_{\alpha}\right\rangle=\sum_{l m} C_{l m} Y_{l m}(\hat{k})
$$

with

$$
\begin{aligned}
C_{l m}= & \sqrt{\frac{\pi}{3}}\left[\left(2 J_{+}+1\right)\left(2 J_{i}+1\right)\left(2 S_{i}+1\right)\right]^{1 / 2} \sum C_{n_{+}^{\prime} n_{+}} C_{n_{i}^{\prime} n_{i}} Q(-1)^{-\Omega_{i}+\Sigma_{i}}\left[1+(-1)^{p}\right] \\
& \times\left[\sum \tilde{I}_{i \lambda \mu ; \Sigma_{e}} G\left(\begin{array}{ccc}
J_{+} & J_{i} & J_{t} \\
-\Omega_{+} & \Omega_{i} & \lambda_{t}
\end{array}\right)\left(\begin{array}{ccc}
S_{+} & S_{e} & S_{i} \\
\Sigma_{+} & \Sigma_{e} & -\Sigma_{i}
\end{array}\right)+\sum \tilde{I}_{l \lambda \mu ; \Sigma_{e}}(-1)^{p_{i}+q_{i}} G\right. \\
& \left.\times\left(\begin{array}{ccc}
J_{+} & J_{i} & J_{t} \\
-\Omega_{+} & -\Omega_{i} & \lambda_{t}
\end{array}\right)\left(\begin{array}{ccc}
S_{+} & S_{e} & S_{i} \\
\Sigma_{+} & \Sigma_{e} & \Sigma_{i}
\end{array}\right)\right]
\end{aligned}
$$

and 


$$
P=\Delta J+\Delta S+2 S_{e}+\Delta p+\Delta q+l+1,
$$

where $\Delta J=J_{+}-J_{i}, \Delta S=S_{+}-S_{i}, \Delta p=p_{+}-p_{i}$, and $\Delta q=q_{+}-q_{i}$, and the $\Sigma$ in Eq. (29) goes over all possible indices. Equations (29) and (30) yield the parity selection rule

$$
\Delta J+\Delta S+2 S_{e}+\Delta p+\Delta q+l=\text { odd }
$$

which is the same as that of Xie and Zare, ${ }^{35}$ and Dixit and McKoy ${ }^{28}$ Note that $\Delta q$ does not arise in the results of Dixit and McKoy, since $\Sigma^{-}$states were not explicitly considered there. For these states the spin-orbit splittings are always negligible and pure Hund's case (b) applies.

In addition to the selection rules of Eqs. (23) and (31), an additional restriction follows from Eqs. (27a) and (27b),

$$
\mu-\lambda= \pm\left(\bar{\Lambda}_{+}+\bar{\Lambda}_{i}\right)
$$

with $\bar{\Lambda}=|\Lambda|$. Equation (23) governs $\bar{\Lambda}_{i} \rightarrow \bar{\Lambda}_{+}$and $-\bar{\Lambda}_{i} \rightarrow-\bar{\Lambda}_{+}$transitions, while Eq. (32) governs $\bar{\Lambda}_{i} \rightarrow-\bar{\Lambda}_{+}$and $-\bar{\Lambda}_{i} \rightarrow \bar{\Lambda}_{+}$transitions. Note that in $\Sigma-\Sigma$ transition, Eq. (23) reduces to Eq. (32).

For pure Hund's case (b) and with the approach of Dixit et al., ${ }^{27,28}$ we can write the photoelectron matrix element of Eq. (28) as

$$
\left\langle f_{\beta}\left|D_{\mu_{0}}\right| i_{\alpha}\right\rangle^{\prime}=\sum_{l m} C_{l m}^{\prime} Y_{l m}(\hat{k})
$$

with

$$
\begin{aligned}
& C_{l m}^{\prime}=\sqrt{\frac{\pi}{3}}\left[\left(2 N_{+}+1\right)\left(2 N_{i}+1\right)\left(2 J_{+}+1\right)\left(2 J_{i}+1\right)\left(2 S_{i}+1\right)\right]^{1 / 2} \sum(-1)^{P^{\prime}}\left(2 N_{t}+1\right) \\
& \times\left(\begin{array}{ccc}
N_{i} & S_{i} & J_{i} \\
M_{i} & M_{S_{i}} & -M_{J_{i}}
\end{array}\right)\left(\begin{array}{ccc}
N_{+} & S_{+} & J_{+} \\
M_{+} & M_{S_{+}} & -M_{J_{+}}
\end{array}\right)\left(\begin{array}{ccc}
S_{+} & S_{e} & S_{i} \\
M_{S_{+}} & M_{S_{e}} & -M_{S_{i}}
\end{array}\right) \\
& \times\left(\begin{array}{ccc}
N_{+} & N_{i} & N_{t} \\
-M_{+} & M_{i} & m_{t}
\end{array}\right)\left(\begin{array}{ccc}
N_{t} & 1 & l \\
-m_{t} & \mu_{0} & -m
\end{array}\right)\left[1+(-1)^{P^{\prime \prime}}\right] \\
& \times\left[\sum \tilde{I}_{i \lambda \mu ; M_{S_{e}}}\left(\begin{array}{ccc}
N_{+} & N_{i} & N_{t} \\
-\Lambda_{+} & \Lambda_{i} & \lambda_{t}
\end{array}\right)\left(\begin{array}{ccc}
N_{t} & 1 & l \\
-\lambda_{t} & \mu & -\lambda
\end{array}\right)\right. \\
& \left.+\sum \tilde{I}_{i \lambda \mu ; M_{S_{e}}}(-1)^{p_{i}}\left(\begin{array}{ccc}
N_{+} & N_{i} & N_{t} \\
-\Lambda_{+} & -\Lambda_{i} & \lambda_{t}
\end{array}\right)\left(\begin{array}{ccc}
N_{t} & 1 & l \\
-\lambda_{t} & \mu & -\lambda
\end{array}\right)\right] \\
& P^{\prime}=M_{+}+M_{J_{+}}-M_{i}+\mu_{0}-N_{i}-N_{+}+S_{i}-\Lambda_{+}-\mu+\frac{1}{2} \text {, }
\end{aligned}
$$

and

$$
P^{\prime \prime}=N_{+}-N_{i}+l+p_{+}-p_{i}+1,
$$

where $N_{+}$and $N_{i}$ are the electronic plus rotational angular momentum of the ionic and resonant states, respectively, $N_{t}$ the angular momentum transfer, and $M_{S_{e}}$ the spin projection along the internuclear axis in the laboratory frame. The generalized vibrationally averaged dipole matrix element $\tilde{I}_{L \lambda \mu: M_{s_{e}}}$ is given as in Eq. (22c) except that its spin component is defined in the laboratory frame. Note that Eq. (33) has been generalized to nonzero spin. Equations (23) and (32) remain the same but the parity selection rule of Eq. (31) becomes

$$
N_{+}-N_{i}+l+p_{+}-p_{i}=\text { odd }
$$

which has been previously derived by Xie and Zare ${ }^{35}$ and Dixit and McKoy. ${ }^{28}$ For a $\Sigma \rightarrow \Sigma$ transition, Eq. (35) further reduces to $N_{+}-N_{i}+l=$ odd. $^{27}$

The differential cross section, Eq. (2), for both coupling schemes can be expressed in terms of Legendre polynomials

$$
\begin{aligned}
\frac{d \sigma}{d \Omega} \propto & \sum_{i} \rho_{i i}\left(\sum_{l m} C_{l m} C_{l^{\prime} m^{\prime}}^{*}{ }_{l^{\prime} m^{\prime}} Y_{l m} Y_{l^{\prime} m^{\prime}}^{*}\right) \\
& =\frac{\sigma}{4 \pi}\left[1+\beta_{2} P_{2}(\cos \theta)+\beta_{4} P_{4}(\cos \theta)+\ldots\right],
\end{aligned}
$$

where $\sigma$ is the total cross section and $\rho_{i i}$ the alignment of $M_{J}$ magnetic levels of the resonant state. To obtain Eq. (36) we used the relationship

$$
\begin{aligned}
Y_{l m} Y_{l^{\prime} m^{\prime}}^{*} \\
=(-1)^{m^{\prime}} \sum_{L, M_{L}}\left[\frac{(2 l+1)\left(2 l^{\prime}+1\right)(2 L+1)}{4 \pi}\right]^{1 / 2} \\
\quad \times\left(\begin{array}{ccc}
l & l^{\prime} & L \\
m & -m^{\prime} & M_{L}
\end{array}\right)\left(\begin{array}{ccc}
l & l^{\prime} & L \\
0 & 0 & 0
\end{array}\right) Y_{L M_{l} \cdot}^{*}
\end{aligned}
$$

Clearly only the $Y_{L, 0}$ terms of Eq. (37) contribute to Eq. (36). 


\section{RESULTS AND DISCUSSION}

As an application of these selection rules for rotationally resolved ion distributions and photoelectron angular distributions, we have studied the $(3+1)$ REMPI of NH via the $3{ }^{3} \Pi$ Rydberg state. The $(3+1)$ REMPI of NH $\left({ }^{3} \Pi \rightarrow{ }^{2} \Pi\right)$ is a good example of the intermediate coupling scheme because $\lambda(\equiv|A / B|)$ lies between Hund's case (a) and (b) in both the resonant and ionic states. For the resonant ${ }^{3} \Pi$ state, ${ }^{47,48} A=36.65 \mathrm{~cm}^{-1}, B=14.34 \mathrm{~cm}^{-1}$, and $\lambda=2.6$; for the ionic $X^{2} \Pi$ state, ${ }^{49} A=77.8 \mathrm{~cm}^{-1}, B=15.35 \mathrm{~cm}^{-1}$, and $\lambda=5.1$. The electronic wave function for the $3^{3} \Pi(3 p \sigma)$ resonant state, with electron configuration $1 \sigma^{2} 2 \sigma^{2} 3 \sigma^{2} 1 \pi 5 \sigma$, was obtained using the improved virtual orbital method ${ }^{50}$ with an extensive Gaussian basis. ${ }^{37}$ The photoelectron orbitals needed in this study were obtained using the iterative Schwinger method in the frozen-core approximation. ${ }^{45}$ The single-center expansion of the $5 \sigma$ orbital around the center of mass gives the following partial wave composition: $12.7 \%$ s, $87.2 \% p$, and $0.2 \% d$ at $R=1.5 a_{0}$, and $85.1 \% s, 5.2 \% p$, and $7.6 \% d$ at $R=3.0 a_{0}$. The rapid orbital evolution gives rise to strong non-Franck-Condon behavior in the vibrational branching ratios, an effect previously predicted to occur in the REMPI of $\mathrm{OH}$ via the analogous $3 p \sigma$ Rydberg state..$^{51,52}$ Further details of these calculations are reported elsewhere. $^{37}$

The multiplet-specific wave functions for the dipole-allowed channels for ionization of the $5 \sigma$ orbital of the Rydberg $3{ }^{3} \Pi$ state of $\mathrm{NH}$ leading to the $X^{2} \Pi$ state of $\mathrm{NH}^{+}$, for $\Sigma_{f}=\left(\Sigma_{+}+\Sigma_{e}\right)=S_{i}=1$, are given by

$\Psi\left({ }^{3} \Pi\right)=\mid($ core $) 1 \pi_{+} k \sigma \mid$,

$\Psi\left({ }^{3} \Delta\right)=\mid($ core $) 1 \pi_{+} k \pi_{+} \mid$,

$$
\begin{aligned}
& \Psi\left({ }^{3} \Sigma^{-}\right) \\
& \quad=\frac{1}{\sqrt{2}}\left[\mid(\text { core }) 1 \pi_{+} k \pi_{-}|-|(\text {core }) 1 \pi_{-} k \pi_{+} \mid\right],
\end{aligned}
$$

and

$$
\begin{aligned}
& \Psi\left({ }^{3} \Sigma^{+}\right) \\
& \quad=\frac{1}{\sqrt{2}}\left[\mid(\text { core }) 1 \pi_{+} k \pi_{-}|+|(\text {core }) 1 \pi_{-} k \pi_{+} \mid\right],
\end{aligned}
$$

with (core) $=1 \sigma^{2} 2 \sigma^{2} 3 \sigma^{2}$. Similar contributions from wave functions for states with $\Sigma_{f}=0,-1$ associated with spin eigenstates $(1 / \sqrt{2})(\alpha \beta+\beta \alpha)$ and $\beta \beta$ must also be included in Eq. (18).

In Fig. 1 we show calculated ionic rotational branching ratios for the $(3+1)$ REMPI via the $N_{11}(22)$ branch of the $3{ }^{3} \Pi, c$ level of the $\left|F_{1}\right\rangle$ state of NH for the diagonal $\left(\Delta v=v_{+}-v_{i}=0\right)$ vibrational bands: $0-0,1-1,2-2$, and $3-3$. We choose this $N_{11}(22)$ branch to facilitate a comparision with the previous study of rotational distributions in REMPI of CH. ${ }^{33}$ The branching ratios are normalized to the most intense transition and convoluted with a Gaussian detector function with a full-width at half-maximum (FWHM) of $6 \mathrm{meV}$. Calculations show that the $c \rightarrow d$ (or $f \rightarrow f$ ) and $\left|F_{1}\right\rangle \rightarrow\left|F_{2}\right\rangle$ transitions are minor (not shown). The ionic rotational distributions are determined for the $N_{11}$ (22) branch because it is a "clean" branch, and it has reached the high- $J$ limit. The rotational branching ratios are slightly asymmetrical around $\Delta N=0$ because the kinetic energies of the photoelectrons for $\Delta N>0$ are smaller than those for $\Delta N<0$. Since $N_{i}=18$, the $\Delta N=0$ peak corre-
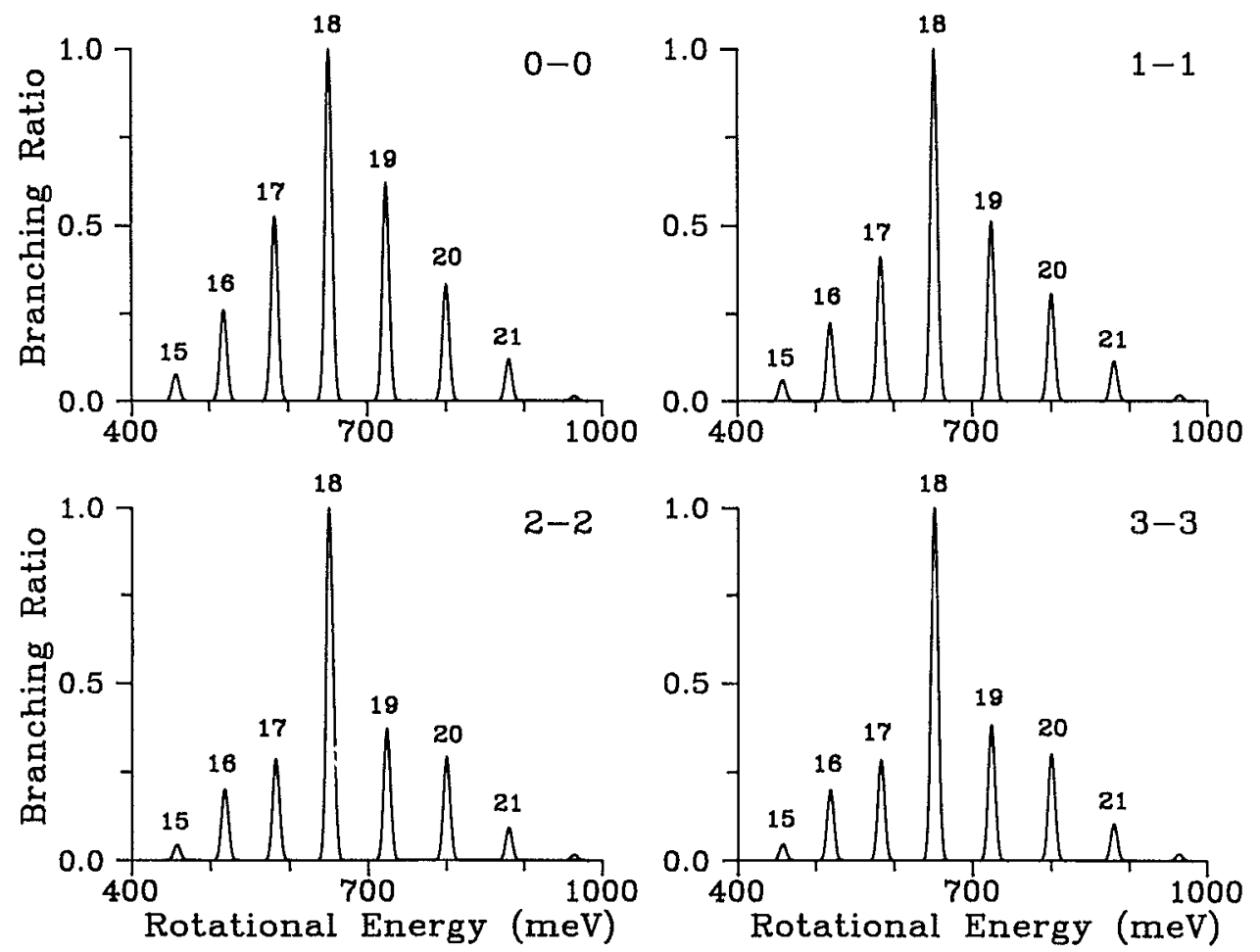

FIG. 1. Ionic rotational branching ratios for the $(3+1)$ REMPI via the $N_{11}$ (22) branch of the $3{ }^{3} \Pi$ state of $\mathrm{NH}$ at the diagonal, $\Delta v=0$, vibrational transition bands. The branching ratios are normalized to the most intense peak and convoluted with Gaussian detector function at FWHM $=6 \mathrm{meV}$. The kinetic energy of photoelectron is $\sim 0.77 \mathrm{eV}$. 

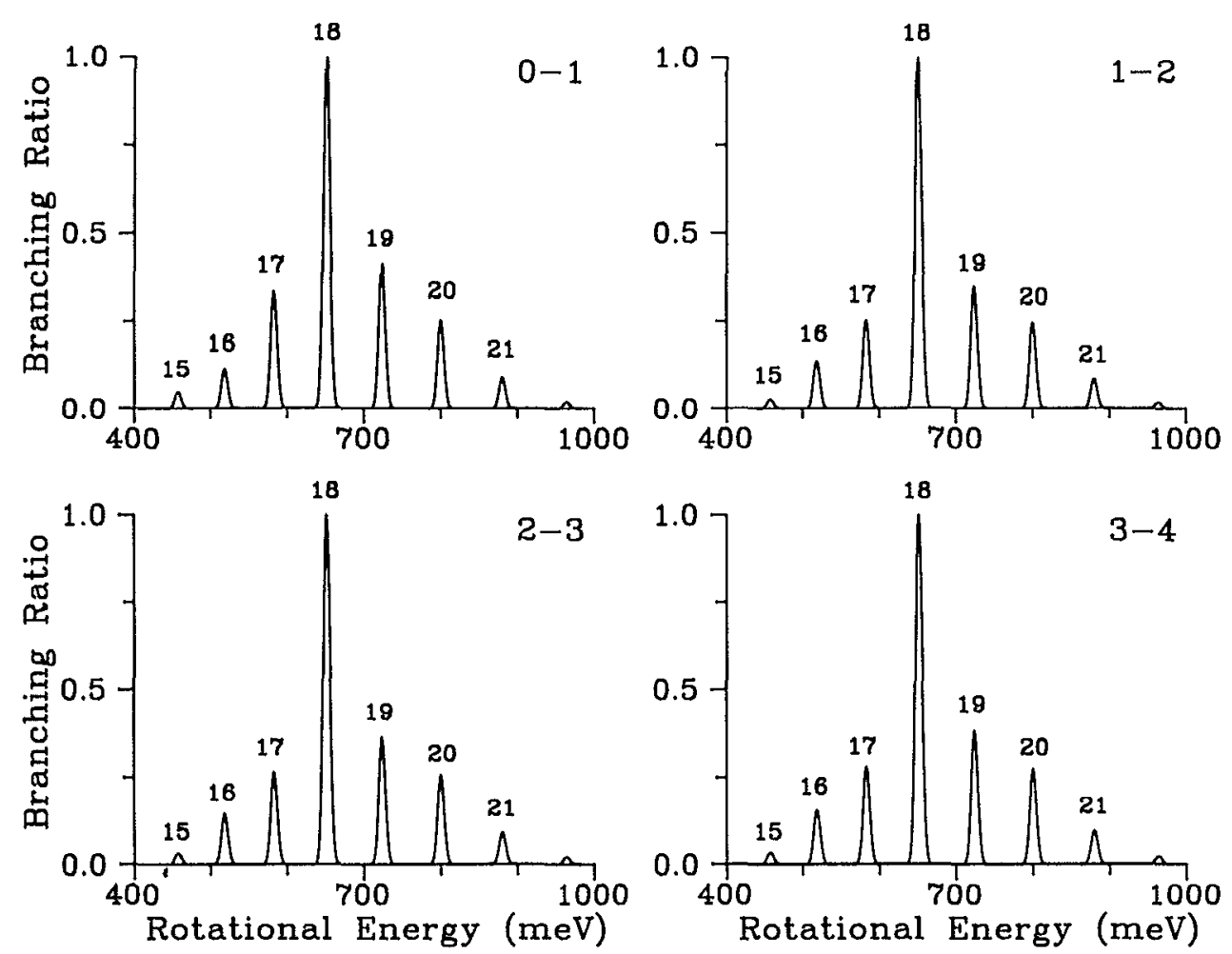

FIG. 2. Same as Fig. 1, but for vibrational transition bands, $\Delta v=1$. The kinetic energy of photoelectron is $\sim 0.38 \mathrm{eV}$.

sponds to $N_{+}=18$. The evolution of the $5 \sigma$ orbital for the $3^{3} \Pi$ state of $\mathrm{NH}$, the $E^{\prime 2} \Sigma^{+}$state of $\mathrm{CH}$, and the $D^{2} \Sigma^{-}$ state of $\mathrm{OH}$ are all similar and rapid as a function of internuclear distance. It changes from predominantly $3 p$ character at small internuclear distances to predominantly $3 s$ character at larger internuclear separations. In contrast to the behavior seen in $\mathrm{CH},{ }^{33}$ we do not see as strong a dependence of rotational distributions on vibrational excitation in the intermediate state. A dominant $\Delta N=$ even distribution of ionic rotational states is predicted in contrast to the $\Delta N=$ odd distribution expected for ionization of a $3 p \sigma$ electron. Simi- lar photoelectron spectra have been measured for the $(2+1)$ REMPI of $D^{2} \Sigma^{-}(3 p \sigma)$ Rydberg state of OH. ${ }^{38}$ Here the Cooper minimum in the $3 p \sigma \rightarrow k \pi(l=2)$ channel $^{37,38,51,52}$ and $l$ mixing in the continuum due to the nonspherical molecular potential have been shown to be responsible for the anomalous distributions. ${ }^{38,53}$ A systematic investigation of the dependence of rotational branching ratios and photoelectron angular distributions on electronic structures of molecular hydrides $\mathrm{CH}, \mathrm{NH}$, and $\mathrm{OH}$ is reported elsewhere. ${ }^{54}$

Figure 2 shows calculated rotational branching ratios

\begin{tabular}{|c|c|c|c|c|c|c|c|}
\hline & \multicolumn{7}{|c|}{$\Delta \mathrm{N}$} \\
\hline Band & -3 & -2 & -1 & 0 & 1 & 2 & 3 \\
\hline $0-0$ & & $\infty$ & & & & 0 & \\
\hline $1-1$ & & 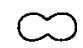 & & & & 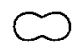 & \\
\hline $0-1$ & & $\infty$ & & & & $\sigma$ & \\
\hline $3-4$ & & $\infty$ & & & & $\infty$ & \\
\hline
\end{tabular}

FIG. 3. Photoelectron angular distributions for the $N_{11}(22)$ branch of the $(3+1)$ REMPI of NH at different $\Delta N\left(\Delta N \equiv N_{+}-N_{i}\right)$ transitions for the $0-0$ and $1-1$ bands of Fig. 1 and the $0-1$ and $3-4$ bands of Fig. 2 . The polarization direction $(\theta=0)$ is vertical. 
for $\Delta v=1$ vibrational bands. We show the $\Delta v=1$ band here since significant ion signals are predicted for this band. ${ }^{37}$ These rotational branching ratios have reached the larger $R$ limit as seen by comparison with those of the $2-2$ and $3-3$ transitions of Fig. 1.

Figure 3 shows rotationally resolved photoelectron angular distributions corresponding to the $0-0$ and $1-1$ bands of Fig. 1 and the $0-1$ and $3-4$ bands of Fig. 2 . Photoelectron angular distributions for other bands are similar to those shown in Fig. 3. The polarization direction $(\theta=0)$ is vertical. Asymmetry parameters up to $\beta_{4}$ in Eq. (36) have been evaluated. The photoelectron angular distributions clearly depend on the rotational state of the ion and are slightly asymmetrical about $\Delta N=0$, due to difference in photoelectron energies. The distributions in Fig. 3 have contributions mainly from $c \rightarrow c$ and $\left|F_{1}\right\rangle \rightarrow\left|F_{1}\right\rangle$ transitions. The photoelectron angular distributions for the $c \rightarrow d$ transition are all polarized perpendicular to the polarization of the incident laser beam and have a relatively small magnitude (not shown). The partial wave contributions to the population of $J^{+}$rotational levels obey the parity selection rule $\Delta N+l=$ odd of Eq. (35) (with $\Delta p=0$ here). Clearly, $s$ and $d$ waves are responsible for the $\Delta N= \pm 1, \pm 3$ transitions and $p$ and $f$ waves for the $\Delta N=0, \pm 2$ transitions. These rotationally resolved photoelectron angular distributions reflect the angular momentum composition of the photoelectron matrix element. Note that the photoelectron angular distributions are plotted assuming $\beta_{0}=1$.

\section{CONCLUSION}

In this paper, we have extended a previous formulation of molecular REMPI photoelectron spectra by explicit inclusion of multiplet-specific final state wave functions and intermediate coupling schemes. We illustrate the utility of this formulation with its application to rotationally resolved photoelectron spectra for $(3+1)$ REMPI of NH via the $3^{3} \Pi$ intermediate state i.e., a ${ }^{3} \Pi \rightarrow{ }^{2} \Pi$ ionizing transition. These photoelectron spectra show an anomalously large $\Delta N=0$ peak which is seen to arise from a Cooper minimum in the $l=2$ component of the $5 \sigma \rightarrow k \pi$ channel. Similar behavior has recently been seen in REMPI photoelectron spectra of $\mathrm{OH}$ via the $D^{2} \Sigma^{-}$Rydberg state. ${ }^{38}$ Experimental ${ }^{36}$ and theoretical studies of the rotationally resolved photoelectron spectra for $(2+1)$ REMPI of NH via the $f^{1} \Pi$, $g^{1} \Delta$, and $h^{1} \Sigma$ Rydberg states are under way and will be reported on elsewhere.

\section{ACKNOWLEDGMENTS}

This work was supported by grants from the National Science Foundation (CHE-8521391), Air Force Office of Scientific Research (Contract No. 87-0039), and the Office of Health and Environmental Research of the U.S. Department of Energy (DE-FG03-87ER60513). We also acknowledge use of resources of the San Diego Supercomputer Center, which is supported by the National Science Foundation, and the resources of the JPL/Caltech CRAY X-MP/18 Supercomputer. K.W. thanks Dr. S. N. Dixit for the useful discussions in the early stage of this work and Dr. J. A. Stephens for the help in obtaining the NH wave functions.

'See, for example, P. M. Johnson and C. E. Otis, Annu. Rev. Phys. Chem. 32, 139 (1981).

${ }^{2}$ J. E. Pollard, D. J. Trevor, J. E. Reutt, Y. T. Lee, and D. A. Shirley, J. Chem. Phys. 77, 34 (1982).

${ }^{3}$ S. T. Pratt, P. M. Dehmer, and J. L. Dehmer, Chem. Phys. Lett. 105, 28 (1984).

${ }^{4}$ M. A. O'Halloran, S. T. Pratt, P. M. Dehmer, and J. L. Dehmer, J. Chem Phys. 87, 3288 (1987).

${ }^{5}$ E. F. McCormack, S. T. Pratt, J. L. Dehmer, and P. M. Dehmer, J. Chem. Phys. 92, 4734 (1990).

${ }^{6}$ S. T. Pratt, E. F. McCormack, J. L. Dehmer, and P. M. Dehmer, J. Chem. Phys. 92, 1831 (1990).

' S. L. Anderson, G. D. Kubiak, and R. N. Zare, Chem. Phys. Lett. 105, 22 (1984).

${ }^{8}$ W. Peatman, F. P. Wolf, and R. Unwin, Chem. Phys. Lett. 95, 453 (1983).

${ }^{9}$ M. A. O'Halloran, P. M. Dehmer, S. T. Pratt, J. L. Dehmer, and F. S Tomkins, J. Chem. Phys. 90, 930 (1989).

${ }^{10}$ S. T. Pratt, P. M. Dehmer, and J. L. Dehmer, J. Chem. Phys. 92, 262 (1990).

${ }^{11}$ W. G. Wilson, K. S. Viswanathan, E. Sekreta, and J. P. Reilly, J. Phys. Chem. 88, 672 (1984).

${ }^{12}$ K. S. Viswanathan, E. Sekreta, E. R. Davidson, and J. P. Reilly, J. Phys. Chem. 90, 5078 (1986)

${ }^{13}$ K. S. Viswanathan, E. Sekreta, and J. P. Reilly, J. Phys. Chem. 90, 5658 (1986).

${ }^{14}$ S. W. Allendorf, D. J. Leahy, D. C. Jacobs, and R. N. Zare, J. Chem. Phys. 91, 2216 (1989).

${ }^{15}$ K. Müller-Dethlefs, M. Sander, and E. W. Schlag, Chem. Phys. Lett. 112, 291 (1984); M. Sander, L. A. Chewter, K. Müller-Dethlefs, and E. W. Schlag, Phys. Rev. A 36, 4543 (1987).

${ }^{16}$ M. Braunstein, V. McKoy, S. N. Dixit, R. G. Tonkyn, and M. G. White, J. Chem. Phys. 93, 5345 (1990).

${ }^{17}$ A. Fujii, T. Ebata, and M. Ito, J. Chem. Phys. 88, 5307 (1988).

${ }^{18}$ J. Xie and R. N. Zare, Chem. Phys. Lett. 159, 399 (1989).

${ }^{19}$ A. D. Buckingham, B. J. Orr, and J. M. Sichel, Philos. Trans. R. Soc. London, Ser. A 268, 147 (1970).

${ }^{20}$ J. M. Sichel, Mol. Phys. 18, 95 (1970).

${ }^{21}$ U. Fano and D. Dill, Phys. Rev. A 6, 185 (1972).

${ }^{22}$ D. Dill, Phys. Rev. A 6, 160 (1972).

${ }^{23}$ M. J. Seaton, Rep. Prog. Phys. 46, 167 (1983).

${ }^{24}$ U. Fano, Phys. Rev. A 2, 353 (1970).

${ }^{25}$ Y. Itikawa, Chem. Phys. 28, 461 (1978); 30, 109 (1978).

${ }^{26}$ N. Chandra, Chem. Phys. 108, 301 (1986).

${ }^{27}$ S. N. Dixit, D. L. Lynch, V. McKoy, and W. M. Huo, Phys. Rev. A 32, 1267 (1985).

${ }^{28}$ S. N. Dixit and V. McKoy, Chem. Phys. Lett. 128, 49 (1986).

${ }^{29}$ H. Rudolph and V. McKoy, J. Chem. Phys. 91, 2235 (1989); H. Rudolph, V. McKoy, and S. N. Dixit, ibid. 90, 2570 (1989); H. Rudolph, S N. Dixit, V. McKoy, and W. M. Huo, ibid. 88, 1516 (1988); S. N. Dixit, D. L. Lynch, V. McKoy, and W. M. Huo, Phys. Rev. A 32, 1267 (1985).

${ }^{30}$ X. Song, E. Sekreta, J. P. Reilly, H. Rudolph, and V. McKoy, J. Chem. Phys. 91, 6062 (1989); H. Rudolph, S. N. Dixit, V. McKoy, and W. M. Huo, ibid. 88, 637 (1988).

${ }^{31}$ H. Rudolph, S. N. Dixit, V. McKoy, and W. M. Huo, Chem. Phys. Lett. 137, 521 (1987).

${ }^{32} \mathrm{~K}$. Wang, V. McKoy, and H. Rudolph (to be published).

${ }^{33}$ H. Rudolph, J. A. Stephens, V. McKoy, and M.-T. Lee, J. Chem. Phys. 91, 1374 (1989).

${ }^{34}$ S. Fredin, D. Gauyacq, M. Horani, C. Jungen, G. Lefevre, and F. Masnou-Seeuws, Mol. Phys. 60, 825 (1987).

${ }^{35}$ J. Xie and R. N. Zare, J. Chem. Phys. 93, 3033 (1990).

${ }^{36}$ E. de Beer, M. Born, C. A. de Lange, and N. P. C. Westwood, Chem. Phys. Lett. (in press).

${ }^{37}$ K. Wang, J. A. Stephens, and V. McKoy, J. Chem. Phys. 93, 7874 (1990). 
${ }^{38}$ E. de Beer, C. A. de Lange, J. A. Stephens, K. Wang, and V. McKoy, J. Chem. Phys. 95, 714 (1991).

${ }^{39} \mathrm{~J}$. B. Halpern, H. Zacharias, and R. Wallenstein, J. Mol. Spectrosc. 79, 1 (1980).

${ }^{40}$ S. N. Dixit, D. L. Lynch, and V. McKoy, in Multiphoton Processes, edited by P. Lambropoulos and S. J. Smith (Springer, Berlin, 1984).

${ }^{41}$ M. Mizushima, The Theory of Rotating Diatomic Molecules (WileyInterscience, New York, 1975).

${ }^{42}$ J. M. Brown, J. T. Hougen, K.-P. Huber, J. W. C. Johns, I. Kopp, H. Lefebvre-Brion, A. J. Merer, D. A. Ramsay, J. Rostas, and R. N. Zare, J. Mol. Spectrosc. 55, 500 (1975).

${ }^{43}$ J. T. Hougen, Natl. Bur. Stand. Monogr. 115, 1 (1970).

${ }^{44}$ A. R. Edmonds, Angular Momentum in Quantum Mechanics (Princeton University, New Jersey, 1974).
${ }^{45}$ R. R. Lucchese, G. Raseev, and V. McKoy, Phys. Rev. A 25, 2572 (1982).

${ }^{46}$ R. R. Lucchese, K. Takatsuka, and V. McKoy, Phys. Rep. 131, 147 (1986).

${ }^{47} \mathrm{H}$. Lefebvre-Brion and R. W. Field, Perturbations in the Spectra of Diatomic Molecules (Academic, Orlando, 1986).

${ }^{48}$ E. M. Goldfield and K. P. Kirby, J. Chem. Phys. 87, 3986 (1987).

${ }^{49}$ R. Colin and A. E. Douglas, Can. J. Phys. 46, 61 (1968).

${ }^{50}$ W. J. Hunt and W. A. Goddard, Chem. Phys. Lett. 3, 414 (1969).

${ }^{51}$ J. A. Stephens and V. McKoy, Phys. Rev. Lett. 62, 889 (1989).

${ }^{52}$ J. A. Stephens and V. McKoy, J. Chem. Phys. 93, 7863 (1990).

${ }^{53}$ K. Wang, J. A. Stephens, and V. McKoy, J. Chem. Phys. (in press).

${ }^{54}$ K. Wang, J. A. Stephens, H. Rudolph, and V. McKoy (to be published). 